\title{
Challenging the gestational age for the limit of viability: proactive care
}

\author{
Stephen Welty ${ }^{1}$
}

Received: 23 October 2018 / Accepted: 29 October 2018 / Published online: 26 November 2018

(c) Springer Nature America, Inc. 2018

In this issue Backes et al. publish work reporting outcomes in patients born between 22 and 22 and 6/7 weeks gestation from two separate institutions using either a "comprehensive approach" or a "selective approach [1]. The definition of comprehensive approach is that all mother infant-dyads were treated with a proactive approach, whereas a selective approach was based on family and physician preferences, one of the three possibilities approaches being a proactive approach. Proactive approaches are described as interventions and care processes focusing on relatively aggressive obstetrical, perinatal, and neonatal approaches to the care of the mothers and neonates, and at 22 weeks gestation they challenge current recommendations. The center using the comprehensive approach was in Uppsala Sweden, Uppsala University Children's Hospital (UUCH) and the center using the selective approach was in Columbus $\mathrm{OH}$, (Nationwide Children's Hospital) (NCH)). They are centers poised to provide innovative care in infants at the edge of viability in that they both admit $>75$ neonates a year under $1500 \mathrm{~g}$ and they have published guideline-driven consistent approaches to the care of infants at the previous "edge of viability", 23 weeks gestation [2, 3].

Publications about how to address approaching neonates in the "gray zone" of size and gestation are relatively plentiful, but, as the authors indicate, what is considered the optimal approach for neonates at very early gestations and extremely low birth weights is controversial. The approach that both centers are willing to provide is not consistent with the most recent recommendations at gestations as early as 22 weeks gestation [4]. Three categories of extremely premature neonates and the approach to the care of these neonates have been described [5]. One category is the gestation and birthweight in which neonates are too

Stephen Welty

stephenwelty@chifranciscan.org

1 University of Washington, Department of Pediatrics, Seattle, USA immature to survive so that aggressive care is not reasonable. Below 23 weeks gestation and under $500 \mathrm{~g}$ has been proposed as defining this category. Another category is one in which there is little controversy about whether initiation of active intervention is reasonable and would include $\geq 25$ weeks gestation and $\geq 600 \mathrm{~g}$. The category in between the two extreme categories has been termed the "gray zone" and the care in this group is a source of active discussion. Recommendations at these gestational ages authored by the American College of Obstetricians and Gynecologists (ACOG), the Society of Maternal Fetal Medicine and the Eunice Kennedy Shriver National Institute of Child Health and Human Development have pushed for clarity in the approaches to neonates at the limit of viability [4]. Neither aggressive obstetrical support (antenatal corticosteroid administration and tocolysis) below 23 weeks gestation, nor "aggressive" newborn resuscitation below 23 weeks gestation is recommended. The recommendations give some leeway for neonatal care at less than 23 weeks based on "individual circumstances" though the definition of individual circumstance is not given. The approach at both $\mathrm{NCH}$ and UUCH challenges these recommendations and as the report indicates they have been providing a more aggressive proactive approach to neonates at 22 weeks gestation for more than a decade.

Despite the limitations of a retrospective analysis of outcomes in the work reported, the report adds significantly to the literature on this topic, and the authors' willingness to address this area of controversy and to compare and report the outcomes using center as a factor analyzed statistically is laudable. Furthermore, over the 10 year duration of the work a total of 56 neonates have been supported with proactive approaches and while this is not a large number on which to base subsequent patient care recommendations the authors point out that the number exceeds those reported in large national studies.

These two centers were poised to add to the literature at 22 weeks gestation as both centers have published reports on neonates at 23 weeks gestation and at that gestation they 
have utilized and described a "proactive" approach with encouraging results. Based on the work at 23 weeks gestation, the two centers are exceptional candidates to implement and analyze similar proactive approaches at 22 gestation.

The higher survival rates and better neurological outcomes at 22 weeks gestational age at UUCH than at NCH is worthy of comments and placing the comments into the context of subtle differences in their respective proactive approaches may provide some basis to speculate about cause and effect. The comparison of all comers between 22 and $0 / 7$ and 22 and $6 / 7$ weeks gestation at both institutions showing a difference in outcomes is not surprising considering that at $\mathrm{NCH}$ proactive care, inconsistent care (proactive obstetrical care and not resuscitated or vice versa), and comfort care are all included in the analyses while at UUCH all patients were treated with proactive care only. However, the different outcomes between centers in the neonates supported with proactive care are intriguing.

The possibilities for these statistically significant differences in outcomes in the proactive approach comparisons include a type I error, in which a difference was detected as a false positive, an inherent difference in the populations of mothers and neonates between centers favoring better outcomes in the UUCH group, and/or a difference in the care processes included under the label of proactive approach.

To provide a context for this discussion, the ACOG has published a series of consensus summaries that have addressed factors associated with neonatal outcomes and they include non-modifiable and potentially modifiable factors. Non-modifiable factors listed by the ACOG include fetal weight, gestational age, and plurality. In the present report there were no differences in these obvious nonmodifiable factors between the centers explaining better outcomes at UUCH. However, there are subtle differences reported suggesting that there are possible non-modifiable factors in which the two populations reported are different. Beyond the obvious difference in geography and ethnic background, additional data from this report suggests that there may be differences in the two populations in that there were 5 of 16 neonates at $\mathrm{NCH}$ that were diagnosed with preeclampsia, and 5 of 16 neonates that were delivered by Cesarean section, whereas in neonates from UUCH there were no sections performed and no patients diagnosed with preeclampsia. These differences suggest that the populations have non-modifiable differences in overt vascular factors (preeclampsia) or subtle vascular factors having detrimental fetal effects that may be more prominent in the $\mathrm{NCH}$ group. This possibility probably warrants further study.

The data contained in this report suggest that the dominant difference between the two centers was in potentially modifiable factors that were subtly different between the two centers proactive care. In the report on the care of neonates at 23 weeks gestation from $\mathrm{NCH}$ proactive management included potentially modifiable obstetrical and neonatal factors, including antenatal steroid administration, Cesarean section for fetal distress, delivery room resuscitation, and neonatal intensive care. Clinicians from UUCH published about their policy for proactive management in 23-25 weeks gestation. Their proactive obstetrical management included administration of antenatal steroids AND tocolysis as well as Cesarean section if indicated. The neonatal management was similar to that described in the $\mathrm{NCH}$ work and in addition "a senior neonatologist" attends all deliveries at 23-25 weeks gestations. Thus, the primary differences in the proactive approaches appear to be the recommendation for tocolysis in the UUCH group and differences in the delivery room management, including more frequent surfactant administration. The differences in outcomes in the present report may reflect the differences in the proactive care provided extrapolated from the management of patients reported at 23 weeks. Important differences are noted in potentially modifiable obstetrical factors in that 30 of 33 mothers received tocolysis at UUCH and 18 of 64 at $\mathrm{NCH}$. In addition, at UUCH 34 of 40 neonates were exposed to two or more doses of antenatal steroids, whereas at $\mathrm{NCH} 4$ of 16 neonates were exposed similarly. Marked differences were also reported in delivery room management and outcomes. The UUCH group had a neonatologist in the delivery room in all deliveries, whereas 12 of 16 deliveries at $\mathrm{NCH}$ had a neonatologist present. At UUCH all neonates received surfactant in the DR and only 1 of 40 needed "active" resuscitation with chest compressions and/ or epinephrine, whereas at $\mathrm{NCH} 9$ of 16 neonates received surfactant in the delivery room and 7 of 16 received "active" resuscitation. These findings suggest that overall differences in outcomes reported resulted for babies leaving the delivery room in better shape at $\mathrm{UUCH}$ than at $\mathrm{NCH}$. This speculation is supported by a marked difference in survival to $24 \mathrm{~h}$ of life between the two centers. Although the details of the resuscitation are not reported beyond cardiopulmonary resuscitation, the findings at $\mathrm{NCH}$ are congruent with previous work published from $\mathrm{NCH}$ finding that cardiopulmonary resuscitation and a "prolonged intubation sequence" defined as needing more than three attempts at intubation were associated with lower survival in their 23 week cohort. It is reasonable to conclude that the differences in survival and neurological outcome were determined by the first few minutes or hours of life, and the subtle differences in the proactive approaches proximal to the first few hours of life are likely to contribute to the differences in outcomes. It is also true that there may also be differences between centers in neonatal intensive care after delivery room care but the details of neonatal care are not described. 
Over the last several decades, improvement in outcomes of extremely premature infants have been largely due to changes in obstetrical care the dominant difference being more frequent utilization of antenatal steroids. The effects of the significant changes in this potentially modifiable factor translated to obstetrical management on outcomes were profound and were largely responsible for a steady decrease in the gestational ages to which the proactive obstetrical, perinatal and neonatal management strategies were considered appropriate.

In the context of subtle changes in proactive care and the associated outcomes, this report is provocative and suggests that at some centers that the definition of gestational age at the lower limit of viability should be challenged. Whereas it is true that the relevant results from this paper are derived from only 56 patients over a 10 year period, there were differences in the outcomes between UUCH and NCH and additional work to refine potentially modifiable factors in the care of these neonates should be done. It is not entirely clear if the differences in outcomes are a result of differences in unrecognized non-modifiable or potentially modifiable factors. However the subtle differences in the obstetrical and perinatal approaches to care of mothers and neonates at 22 weeks gestation and the outcomes reported suggest strongly that potentially modifiable factors contribute to improved outcomes in neonates at 22 weeks and that comparative effectiveness research on proactive approaches should be done. Furthermore, the proactive care approaches described in this report include only details of obstetrical and delivery room care and approaches in neonatal intensive care after the delivery room are not discussed, but should probably only be studied after best obstetrical and delivery room management strategies are defined.

\section{References}

1. Backes C, Söderström F, Ägren J, Sindelar R, Bartlett C, Rivera B, et al. Outcomes following a comprehensive versus selective approach for infants born at 22 weeks of gestation. JPER JPER. 2018; https://doi.org/10.1038/s41372-018-0248-y.

2. Backes C, Rivera B, Haque U, Srouji L, Beck S, Yin H, et al. A proactive approach to neonates born at 23 weeks of gestation. Obstet Gynecol. 2015;126:939-46.

3. Häkansson S, Farooqi A, Holmgren P, Serenius F, Högberg U. Proactive management promotes outcome in extremely preterm infants: a population-based comparison of two perinatal management strategies. Pediatrics. 2004;114:58-64.

4. Raju T, Mercer B, Burchfield D, Joseph G Jr. Periviable birth. Obstet Gynecol. 2014;123:1083-95.

5. Seri I, Evans J. Limits of viability: definition of of the gray zone. J Perinatol. 2008 Suppl 1:S4-8. 